\title{
Impairment-Aware Routing and Waveband Assignment for Efficient Optical Transport Networks
}

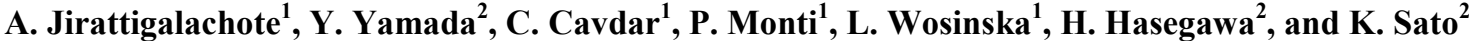 \\ ${ }^{I}$ Royal Institute of Technology KTH, Isafjordsgatan 22, Electrum 229, 16440 Kista, Sweden \\ ${ }^{2}$ Nagoya University, Furo-cho, Chikusa-ku, Nagoya, 464-8603, Japan \\ \{amornrat, cavdar,pmonti,wosinska\}@kth.se,yo_yamada@echo.nuee.nagoya-u.ac.jp, \{hasegawa, sato\}@nuee.nagoya-u.ac.jp
}

\begin{abstract}
We demonstrate the importance of considering physical impairments in wavebandbased optical networks. We propose and evaluate an Impairment-Aware waveband-routing and waveband-assignment (IA-RWBA) algorithm achieving significant improvement in both impairment satisfaction rate and total network cost.

OCIS codes: (060.0060) Fiber optics and optical communications; (060.4250) Networks
\end{abstract}

\section{Introduction}

With the rapid growth of traffic demand, transport networks are evolving towards more aggregated and dense switching architectures. In such a scenario optical networks based on wavebands (WBs) are expected to play a key role since they can offer reduction of the complexity and the size of optical switching fabrics. A waveband is defined as a bundle of optical wavelength paths (lightpaths) that are grouped and switched together using a single end-to-end or a concatenation of multiple WBs. Introduction of hierarchical optical switching architectures which jointly incorporate waveband and wavelength switching technologies leads to significant gain in overall optical switching cost and complexity by reducing the total number of optical switch ports [1]. In a transparent optical network, where optical paths and wavebands are transmitted without any regeneration, quality of optical signals may suffer from the effect of accumulated physical layer impairments. Once optical paths are grouped into a waveband, regeneration is not an option unless the waveband is terminated and processed electronically. There are two important impairments to consider in a waveband routed network; $(i)$ presence of an exacerbated crosstalk effect due to having a bundle of optical paths that are grouped and switched together in the same waveband, and (ii) the extra attenuation that the optical signal has to experience while traversing a series of waveband selective switches. In conventional wavelength routed networks, physical layer impairments are considered during the provisioning phase by solving the Impairment-Aware Routing and Wavelength Assignment (IA-RWA) problem. Despite of the fact that the issue of physical layer impairments is addressed in a vast amount of literature, a little work has been done so far to solve the Impairment-Aware RWBA (IA-RWBA) problem. In [2] authors consider the impact of the optical signal attenuation on the overall performance of two waveband grooming strategies, i.e., Dedicated Bandpath Strategy (DBS), and Shared Bandpath Strategy (SBS). The authors in [3] try to mitigate the effect of optical signal impairments by introducing regeneration in a waveband switched network. With this objective in mind they devise an efficient method to minimize the number of regenerators that needed to be placed. However, using regenerators that are expensive components may negatively impact the cost savings achieved in a waveband switched network. Moreover, the authors in [2,3] consider only the Amplified Spontaneous Emission (ASE) noise and do not take into account the effect of other impairments, i.e., crosstalk, which are in particular important in waveband switched networks. Furthermore, in [2] physical layer impairments are considered a-posteriori and they are not taken into account during the route computation phase. In this study we propose an IA-RWBA algorithm which takes into account physical layer impairments in the routing and waveband assignment phase without the requirement of regenerators.

Our IA-RWBA algorithm is applied to the hierarchical optical cross-connect (HOXC) architecture proposed in [4]. The waveband add/drop ratio of a HOXC is defined as the ratio of added/dropped waveband paths to all outgoing/incoming wavebands from/to the waveband cross-connect. The waveband add/drop ratio can be divided into two categories, namely originating/terminating and grooming. In this context grooming is related to aggregating the wavelengths passing through the node into the wavebands, using wavelength cross-connect part of HOXC. In [4], the proposed HOXC architecture can substantially reduce the node switch scale by limiting grooming capability, while the network cost is dramatically reduced by the proposed RWBA algorithm with small amount of grooming hardware. However, the proposed RWBA does not consider the physical layer impairments that need to be addressed to guarantee an appropriate signal quality.

In our IA-RWBA, the effect of physical layer impairments, namely Polarization Mode Dispersion (PMD), ASE noise, and crosstalk, is considered during the network planning phase. Simulation results on a realistic European test 
network topology show that our proposed algorithm not only achieves significant improvement in terms of impairment satisfaction rate, i.e., the percentage of connection requests with signal quality better than the BER threshold, compared to the algorithm in [4], but also drastically reduces the network cost, compared to the End-toEnd scheme which combines lightpath requests into wavebands that can be routed through the shortest paths between the source and destination nodes of each lightpath.

\section{Impairments Model}

In this work, the effect of physical layer impairments is quantified by using the modified quality factor Q from [5]. Q-factor includes PMD, ASE noise, and crosstalk at multiplexers/de-multiplexers and switches. The power penalty due to PMD is calculated based on the length of lightpath/waveband path, bit rate, and PMD parameter. ASE and crosstalk are calculated assuming that they follow a Gaussian distribution. The Q-factor of a lightpath/waveband path is given according to $Q=P_{\text {transmitter }} /\left(\operatorname{pen}_{P M D} \cdot \sqrt{\sigma_{A S E}^{2}+\sigma_{X T}^{2}}\right)$, where $P_{\text {transmitter }}$ is the power of transmitted signal, $\operatorname{pen}_{P M D}$ is the power penalty due to PMD [6], $\sigma_{A S E}^{2}$ is the electrical variance of ASE noise, and $\sigma_{X T}^{2}$ is the electrical variance of crosstalk induced in multiplexers/de-multiplexers and switches [7]. The BER value of a lightpath/waveband path can be derived from $B E R=0.5 \cdot \operatorname{erfc}(Q / \sqrt{2})$.

\section{Algorithm Description}

We propose a new IA-RWBA with the objective to minimize the total network cost and to attain the given BER requirement of each optical path as much as possible. To find a group of traffic demands whose source nodes (or destination nodes) are close to each other in order to groom them into a waveband path, the algorithm in [4] adopts a source-destination (s-d) Cartesian product space to easily evaluate the closeness of source/destination node pairs. In such space, the nearby connection requests are classified as clusters of points.

By taking advantage of the significant network cost reduction achieved by the algorithm in [4], our proposed approach modifies the criterion to identify a cluster in [4] in order to take into account the effect of physical layer impairments as follows. First, the BER value of the longest route out of shortest paths between all the node pairs is calculated using the expressions provided in the previous section under the assumption that all the network resources are in use. This BER value is then used as the BER threshold $\left(B E R_{t h}\right)$ while identifying a cluster. During the search to determine the source/destination nodes to be included in a cluster, the BER of a route between a source/destination pair considering only ASE noise and PMD penalty (i.e., parameter $\sigma_{X T}^{2}$ is set to zero), $B E R(s, d)_{A S E, P M D}$, is calculated. In order to quantify the effect of crosstalk on the optical signal quality, the information regarding signal grooming is needed. Thus, in this step the BER margin $\left(B E R_{\text {margin }}\right)$ considering the possible impact of crosstalk is set. Source and destination nodes are included in clusters if and only if $\left(B E R(s, d)_{A S E, P M D} \cdot B E R_{\text {margin }}\right)$ is less than or equal to $B E R_{t h}$. Otherwise, the connection requests are established in the last step of algorithm, while accommodating the remaining connection requests. After identifying a cluster, wavebands are then established and the corresponding lightpaths in the retrieved cluster are accommodated. Finally, the algorithm considers the remaining connection requests by leveraging on the unused capacity of existing waveband paths. In the case where $\left(B E R(s, d)_{A S E, P M D} \cdot B E R_{\text {margin }}\right)$ of a remaining connection request is greater than $B E R_{t h}$, the number of grooming operation is reduced or the alternative route computed by using Dijkstra's algorithm is used instead.

\section{Numerical Results}

For the performance evaluation our IA-RWBA is compared to Impairment-Unaware RWBA (IUa-RWBA) proposed in [4], together with the End-to-End scheme as a benchmark. The Pan-European test network topology (COST239) [5] which comprises of 11 nodes and 26 bidirectional fiber links is used. We assume randomly and uniformly distributed traffic demands represented as the average number of connection requests between each node pair. For each of the tested traffic demands, 100 different random traffic distributions are considered and averaged results are plotted. Two different fiber capacities are tested, (i) 40 wavelengths: 8 wavelengths/waveband with 5 wavebands/fiber (W8B5), and (ii) 80 wavelengths: 8 wavelengths/waveband with 10 wavebands/fiber (W8B10). The transmission link model in [5] and the HOXC architecture presented in [4] are used. In our study, the effect of physical layer impairments is quantified in terms of impairment satisfaction rate, defined as the percentage of connection requests with signal quality better than the BER threshold, i.e., $10^{-9}$. Furthermore, we evaluate the total network cost which is the sum of the node and link cost. The node cost includes the number of optical switch ports 
and a constant value for the control system, while the link cost consists of the cost of optical fibers and amplifiers placed in fixed interval. See [8] for details.

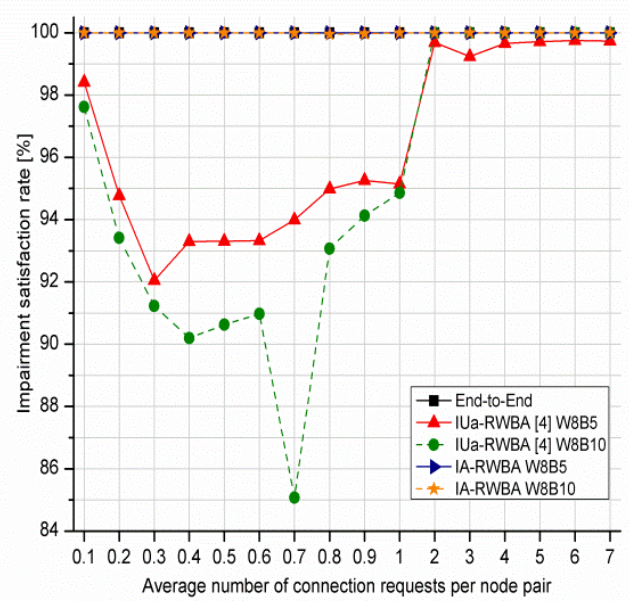

Fig. 1. Impairment satisfaction rate vs. average number of connection requests per node pair

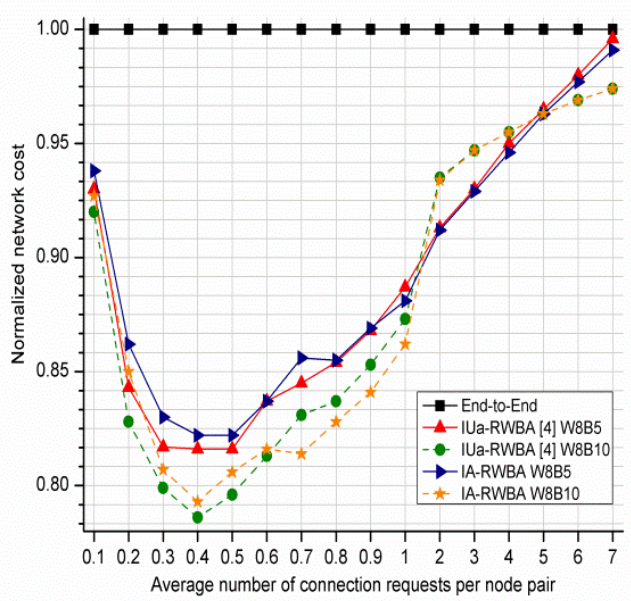

Fig. 2. Normalized network cost vs. average number of connection requests per node pair

Fig. 1 presents the impairment satisfaction rate as a function of the average number of connection requests per node pair. It can be seen clearly that with IUa-RWBA up to $15 \%$ of connection requests cannot meet the required BER threshold. This is because IUa-RWBA takes longer routes in order to groom larger number of optical paths into wavebands to reduce the number of switching ports. On the other hand $100 \%$ impairment satisfaction rate can be achieved by using our IA-RWBA algorithm having an additional intelligence in considering the optical reach of the paths and crosstalk. For the average number of connection requests per node pair larger than 2 all algorithms are able to achieve a certain level of signal quality. It is because with the increasing load the flexibility of waveband assignment is decreasing and consequently, the benefit of our algorithm is reduced.

Fig. 2 shows the total network cost obtained by the IUa-RWBA and IA-RWBA algorithms, normalized according to the End-to-End scheme. IA-RWBA achieves cost savings up to $18 \%$ and $21 \%$ in the case of W8B5 and W8B10, respectively. It is important to note that our IA-RWBA algorithm is able to not only guarantee the quality of optical signal but also provide a significant cost saving, close to IUa-RWBA.

\section{Conclusions}

We study the impact of physical layer impairments on the performance of a waveband routed optical network, which is necessary to maximize the benefits of transparent waveband routed optical networks that refrain from using costly regeneration. In order to mitigate the effect of physical layer constraints, we propose an IA-RWBA algorithm for a given set of lightpath requests which takes into account the effect of impairments, namely PMD, ASE noise, and crosstalk, in the routing and waveband assignment phase. Simulation results show that our IA-RWBA algorithm can offer $100 \%$ impairment satisfaction rate in a realistic European scale network without need of optical signal regeneration, while the offset of cost is minimal compared to IUa-RWBA, and as a result significant saving in the total network cost is retained.

\section{Acknowledgment}

The research leading to these results was supported by Optical Networking Systems (ONS) focus projects, part of ICT The Next Generation (TNG) Strategic Research Area (SRA) initiative at the Royal Institute of Technology.

\section{References}

[1] K. Sato et al., IEEE/OSA Journal of Communications and Networking, vol. 1, no. 2, pp. A81-A93, Jul. 2009.

[2] H. H. Yen et al., in Proc. of IEEE/OSA OFC/NFOEC2007, Mar. 2007.

[3] S. Varma et al., in Proc. of IEEE ICC2011, Jun. 2011.

[4] Y. Yamada et al., in Proc. ECOC2010, Sep. 2010.

[5] A. Jirattigalachote et al., Journal of Networks, Special Issue on All-Optically Routed Networks, vol. 5, no. 11, pp. 1279-1289, Nov. 2011

[6] A. Jirattigalachote et al., in Proc. of IEEE/OSA/SPIE ACP2009, Nov. 2009.

[7] B. Ramamurthy et al., IEEE Journal of Lightwave Technology, vol. 17, no. 10, pp. 1713-1723, Oct. 1999.

[8] I. Yagyu et al., IEEE Journal on Selected Areas in Communications, vol. 26, no. 6, Part Supp., pp. 22-31, Aug. 2008. 